\title{
Survival in Patients with Ischaemic Cardiomyopathy and Ventricular Tachyarrhythmias: Role of Catheter Treatment
}

\section{Garkina S*, Tatarskiy R and Lebedev D}

Almazov National Medical Research Centre, Saint Petersburg, Russian Federation

*Corresponding author: Svetlana Garkina, Almazov National Medical Research Centre, Saint Petersburg, Russian Federation, Tel: +7812702-37-06; E-mail: svetlanagarkina@yahoo.com

Received: July 20, 2018; Accepted: August 23, 2018; Published: August 28, 2018

Copyright: (C) 2018 Garkina S, et al. This is an open-access article distributed under the terms of the creative commons attribution license, which permits unrestricted use, distribution, and reproduction in any medium, provided the original author and source are credited.

\begin{abstract}
Introduction: The last decades demonstrated significant progress in catheter treatment techniques, with several theories and findings presenting the detailed knowledge of the substrates and patterns of ventricular tachyarrhythmias with structural heart diseases. It is still an actively developing method of treatment requiring detailed assessment including electrical storm.
\end{abstract}

Study objectives: To estimate efficacy and survival in patients with ischaemic cardiomyopathy and ventricular tachyarrhythmias undergoing catheter ablation.

Materials and Methods: Study enrolled patients with prior myocardial infarction and documented episodes of sustained ventricular tachyarrhythmia (within not less than 40 days after acute myocardial infarction) registered on ECG, 24 hours Holter monitor or during ICD programming and/or ICD telemetry. We evaluated 72 patients (mean age of $64 \pm 13$ years, 63 males) with postinfarction ventricular tachyarrhythmias. Among them there were 12 patients with emergent catheter ablation of electrical storm.

Results: During $35 \pm 16$ months of follow-up period $63 \%$ of patients didn't present any sustained ventricular tachyarrhythmias. Perioperative fatal outcomes were not registered. The implemented strategy did not deteriorate left ventricle ejection fraction after catheter treatment according to control echocardiography data $(30 \pm 13 \%$ vs $29 \pm$ $11 \%$ after RFA, $p>0.05)$. In patients with catheter ablation of electrical storm during the first year of follow-up the recurrent ventricular tachycardias were registered in $33 \%$ of cases (4 patients). In single case the rare paroxysms were effectively stopped by ICD anti-tachycardia pacing without electrical storm. During follow-up period the recurrent electrical storms were not registered, general mortality rate from progressing heart failure was $25 \%$ (3 patients) in the first 18 months after catheter treatment. Acute and long-term efficacy of electrical storm elimination was equal to $100 \%$, whereas complete reduction of arrhythmias during the long-term period was achieved in $75 \%$ of patients (including repeated RFA sessions). Most of patients (67\%) presented clinically relevant heart failure with high functional class (NYHA III-IV) at the moment of electrical storm and 6 of 8 patients then had significant improvement after catheter ablation.

Conclusion: During 5 years of follow up the overall efficacy of catheter elimination of ventricular tachyarrhythmias and patients' survival was $63 \%$ and $78 \%$ respectively. The was no statistically significant difference in mortality rate in patients with effective catheter treatment and those with recurrent ventricular tachyarrhythmia after RFA.

Keywords: Ventricular tachyarrhythmias; Catheter ablation; Electrical Storm; Structural heart diseases

\section{Introduction}

The last decades demonstrated significant progress in radiofrequency ablation (RFA) field, with several theories and findings presenting the detailed knowledge of the substrates and patterns of ventricular tachyarrhythmias with structural heart diseases. Moreover, the development of irrigated ablation catheters, complex mapping systems, methods of epicardial mapping and ablation, advanced instruments for hemodynamic support and assistance of computed tomography/magnetic resonance imaging improved the clinical success and reduced the perioperative complications. The progress of the modern catheter ablation technology resulted in further change of the ablation role in clinical guidelines.

Ventricular arrhythmias are the frequent cause of mortality in patients with ischaemic cardiomyopathy [1]. The high mortality risk can be lessened, according to investigations' data, by implantation of cardioverter defibrillator (ICD), but it still has significant limitations because none of the appliances provides complete protection from lifethreatening arrhythmias [2]. Although ICDs prolong the survival, the frequent electrotherapy is associated with decreased quality of life, hospitalization and increased mortality [3].

The catheter ablation and antiarrhythmic drugs are used for reducing the ICD interventions rate and potentially must improve survival in patients with ventricular tachyarrhythmia [4,5]. The antiarrhythmic drugs have not provided the survival improvement 
Page 2 of 6

along with optimal reducing of ICD shocks, but also revealed serious side effects [3]. The recent study VANISH (Ventricular tachycardia ablation versus escalation of antiarrhythmic drugs) demonstrated the lower frequency of the composite primary endpoint in patients with ischaemic cardiomyopathy, ICD and re-entry ventricular tachycardias, in spite of the antiarrhythmic drug usage. Reduction of mortality, occurrence of electrical storm or ICD shocks in patients undergoing catheter ablation is evident comparing with antiarrhythmic drug escalation [6]. In spite of the technological innovation in the field of ventricular tachyarrhythmia catheter ablation, it is still an actively developing method of treatment requiring detailed assessment including electrical storm.

\section{Study Objectives}

To estimate efficacy and survival in patients with ischaemic cardiomyopathy and ventricular tachyarrhythmias undergoing catheter ablation.

\section{Materials and Methods}

Study enrolled patients with prior myocardial infarction (MI) and documented episodes of sustained ventricular tachyarrhythmia (within not less than 40 days after acute MI) registered on ECG, 24 hours Holter monitor or during ICD programming and/or ICD telemetry.

We evaluated 72 patients (mean age of $64 \pm 13$ years, 63 males) with postinfarction ventricular tachyarrhythmias. Among them there were 12 patients with emergent catheter ablation of electrical storm. All patients were divided in two main groups depending on tachyarrhythmia recurrence after ablation during follow up period. First group consisted of 27 patients (37\%) with recurrent ventricular tachyarrhythmias after RFA, mean age $62 \pm 10$ years. Second group included 45 patients $(63 \%)$ without recurrent tachyarrhythmia, mean age $63 \pm 12$ years. In our study most patients were males, which can be explained by the fact that ischemic heart disease (IHD) is mostly spread in male population. Average time of ventricular tachycardia manifestation was $13 \pm 9$ years after MI. The characteristics of the groups of patients are presented in Table 1.

Myocardial reperfusion (percutaneous intervention and/or coronary artery bypass grafting) was indicated to 60 patients. Due to the long time passed from acute myocardial infarction the frequency of thrombolysis could not be defined, that is why this factor was not evaluated as a predictor of the risk of ventricular tachyarrhythmia recurrence. The main reason for nonperformance of myocardial reperfusion was chronic coronary arteries occlusion.

As shown in the table, the main clinical predictors of postinfarction ventricular tachycardias recurrence after ablation were the long period from acute MI and its anterior location.

The planned monitoring protocol included the regular clinical assessment and analysis of ICD monitoring either manual or using the remote monitoring. Unscheduled visits took place in case of ventricular tachyarrhythmia. ICD telemetry included the ventricular tachyarrhythmia analysis (frequency, duration), electrotherapy type and adequacy. Transthoracic echocardiography, regular ECG registration and 24 hours Holter monitoring were also performed in all cases. The average follows up period was five years.

The mapping procedure and the following radiofrequency catheter destruction were divided in several stages (Figure 1). In brief, the procedure included substrate-oriented catheter ablation of all conducting channels, fractionated and late potentials (till their disappearance). Mapping and ablation were performed using LabSystem PRO (EP Recording Systems, Bard, USA), CardioLab IT (GE Medical Systems, USA) and non-fluoroscopic electroanatomical mapping system CARTO XP or CARTO 3 (Biosense Webster, USA) with irrigated catheters (power up to $45 \mathrm{~W}$, maximum temperature $43^{\circ} \mathrm{C}$ and irrigation flow rate of $17-30 \mathrm{ml} / \mathrm{min}$ ).

\begin{tabular}{|c|c|c|c|c|}
\hline Characteristic & Total 72 & I group 27 (37\%) & II group 45 (63\%) & Validity \\
\hline Age, years & $64 \pm 13$ & $62 \pm 10$ & $63 \pm 12$ & $p>0.05$ \\
\hline Male, n (\%) & $63(88)$ & $25(93)$ & $38(85)$ & $p>0.05$ \\
\hline Diabetes, n (\%) & $11(15)$ & $3(11)$ & $8(18)$ & $p>0.05$ \\
\hline Atrial Fibrillation/Atrial Flutter, $\mathrm{n}(\%)$ & $14(19)$ & $6(22)$ & $8(18)$ & $p>0.05$ \\
\hline Time after acute myocardial infarction, years & $13 \pm 9$ & $9 \pm 6$ & $14 \pm 10$ & $p \leq 0.05^{*}$ \\
\hline Anterior myocardial infarction, $\mathrm{n}(\%)$ & $43(60)$ & $19(70)$ & $24(53)$ & $p \leq 0.05^{*}$ \\
\hline Inferior myocardial infarction, $\mathrm{n}(\%)$ & $29(40)$ & $11(40)$ & $18(40)$ & $p>0.05$ \\
\hline Multiple infarctions, $n(\%)$ & $11(15)$ & $3(11)$ & $8(18)$ & $p>0.05$ \\
\hline Myocardial reperfusion & $60(83)$ & $19(70)$ & $41(91)$ & $p>0.05$ \\
\hline \multicolumn{5}{|l|}{ Heart Failure functional class (NYHA), n (\%) } \\
\hline 1 & $22(31)$ & $7(26)$ & $15(33)$ & $p>0.05$ \\
\hline ॥ & $34(47)$ & $14(52)$ & $20(44)$ & $p>0.05$ \\
\hline III or IV & $16(22)$ & $6(22)$ & $10(22)$ & $p>0.05$ \\
\hline
\end{tabular}


Page 3 of 6

\begin{tabular}{|c|c|c|c|c|}
\hline LV EF, \% & $30 \pm 13$ & $28 \pm 11$ & $33 \pm 16$ & $p>0.05$ \\
\hline Left ventricle aneurysm, n (\%) & $20(28)$ & $7(26)$ & $13(29)$ & $p>0.05$ \\
\hline \multicolumn{5}{|l|}{ Antiarrhythmic drug, $\mathrm{n}(\%)$} \\
\hline Amiodarone & $42(58)$ & $18(67)$ & $24(53)$ & $p>0.05$ \\
\hline Sotalol & $0(28)$ & $6(22)$ & $14(31)$ & $p>0.05$ \\
\hline Beta-blockers & $63(88)$ & $24(89)$ & $39(87)$ & $p>0.05$ \\
\hline ICD/CRT-D, n (\%) & $46(64)$ & 18 & 28 & $p>0.05$ \\
\hline
\end{tabular}

Table 1: Clinical characteristics of the patients.

The usage of non-fluoroscopic system makes it possible to identify late potentials as digitals, so the local activation value on mapping lead was set to the second spike of abnormal electrogram. Thus, during the left ventricular reconstruction the pathological area corresponded to the later activation in comparison with the reference lead and was presented in violet color (Figure 2). In this case the late potentials were defined as activation area more than $38 \mathrm{~ms}$ after QRS complex. Beside the voltage characteristics of the patient (on the right) the pathological potentials were found mostly inside and in peri-infarction zone. The arrows on the electrogram mark the double potentials.

The final stage after the extended catheter ablation was the repeated stimulation protocol for ventricular tachyarrhythmia induction with the same parameters as before the ablation. Some of patients ( 7 patients) had not undergone the stimulation protocol because of the heart failure progression and deteriorated hemodynamic status. Also the stimulation protocol was not performed in 5 patients because of the multiple electrical cardioversions during RFA.

After RFA procedure full stimulation protocol for ventricular tachycardia induction was applied to 60 patients. In $68 \%$ of cases (41 patients) the ventricular tachyarrhythmias were not provoked. Sustained ventricular tachycardia was inducted in 8 patients whereas 11 patients presented transient unmappable ventricular tachycardias. Mappable ventricular tachycardia was defined as hemodynamically stable and monomorphic with sustained cycle length. It stands to mention that even longer cycle of tachycardia can make negative impact on the hemodynamics in patients with high functional class of chronic heart failure.

\section{Results and Discussion}

During $35 \pm 16$ months of follow-up period $63 \%$ of patients didn't present any sustained ventricular tachyarrhythmias. Perioperative fatal outcomes were not registered. The implemented strategy did not deteriorate left ventricle ejection fraction after catheter treatment according to control echocardiography data $(30 \pm 13 \%$ vs $29 \pm 11 \%$ after RFA, $p>0.05$ ). In patients with catheter ablation of electrical storm during the first year of follow-up the recurrent ventricular tachycardias were registered in $33 \%$ of cases (4 patients).

In single case the rare paroxysms were effectively stopped by ICD anti-tachycardia pacing without electrical storm. During follow-up period the recurrent electrical storms were not registered, general mortality rate from progressing heart failure was $25 \%$ (3 patients) in the first 18 months after catheter treatment.

Initially the significant decrease of left ventricle ejection fraction was registered in these patients. Later on, three patients underwent repeated catheter ablation procedures; in two of them the rare paroxysms of ventricular tachycardia initially estimated of epicardial location had remained, in the third case ventricular tachyarrhythmias were not registered after catheter treatment.

Acute and long-term efficacy of electrical storm elimination was equal to $100 \%$, whereas complete reduction of arrhythmias during the long-term period was achieved in $75 \%$ of patients (including repeated RFA sessions). Most of patients (67\%) presented clinically relevant heart failure with high functional class (NYHA III-IV) at the moment of electrical storm and 6 of 8 patients then had significant improvement after catheter ablation.

The overall efficacy of catheter treatment of post-infarction ventricular tachyarrhythmia during long follow-up period (KaplanMeier curve) is represented on Figure 3.

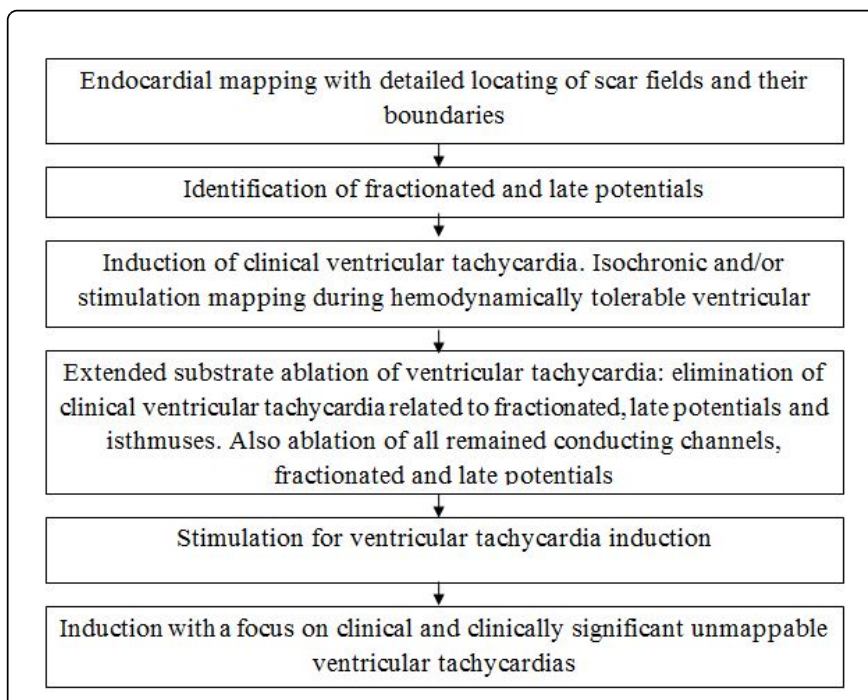

Figure 1: Stages of ventricular tachyarrhythmia catheter destruction. 
Citation: Garkina S, Tatarskiy R, Lebedev D (2018) Survival in Patients with Ischaemic Cardiomyopathy and Ventricular Tachyarrhythmias: Role of Catheter Treatment. J Cardiovasc Dis Diagn 6: 336. doi:10.4172/2329-9517.1000336

Page 4 of 6

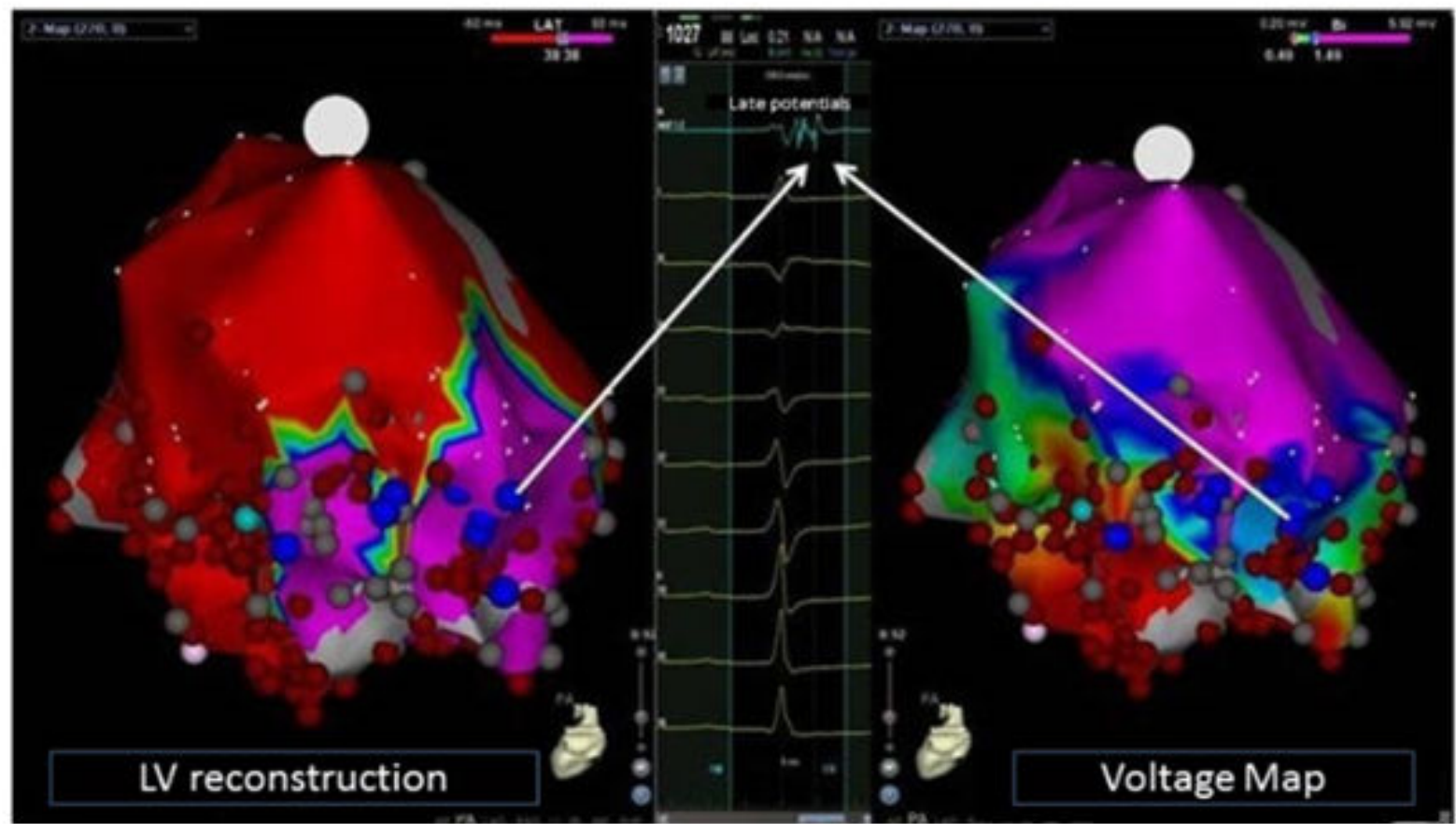

Figure 2: LV reconstruction.

According to the Kaplan-Meier curve data, the recurrent tachyarrhythmias were mostly registered during the first 30 months of follow-up. The further follow-up revealed the steady course with minimum number of recurrent ventricular tachyarrhythmia events.

Only in 5 patients the multiple ventricular tachycardias after ablation took place, but in the early postoperative period. During the 5 years of the follow-up the total mortality was $22 \%$ (16 patients).

Among them 3 patients with clinically relevant heart failure died during in hospital period, while progressing multisystem failure was the cause of death in two cases. That is why it is unlikely that these adverse outcomes were associated with the catheter treatment.

During the study period there were also registered 4 cases of continuously recurrent ventricular fibrillation mortality based on remote ICD monitoring data, 3 fatal pulmonary embolism episodes while in 6 cases the cause of death was unknown. The survival rate after catheter treatment of post-infarction ventricular tachyarrhythmias is presented on Figure 4.

The analysis of the Kaplan-Meier curve shows that most of fatal cases were registered during the first 2 years after RFA procedure, then the course was stable up to 5 years.

If compared the survival in both groups - patients with successful ablation and patients with recurrent ventricular tachyarrhythmias there was no statistically significant difference during the 5 years of study (Figure 5).

Now-a-days catheter ablation has become the integral part of ventricular tachyarrhythmia treatment with the adequate efficacy and relatively low risk. According to our results catheter treatment of recurrent ventricular tachyarrhythmias in patients with underlying
IHD contributed to sufficient acute effect and sustained long-term efficacy during 5 years with acceptable perioperative safety.

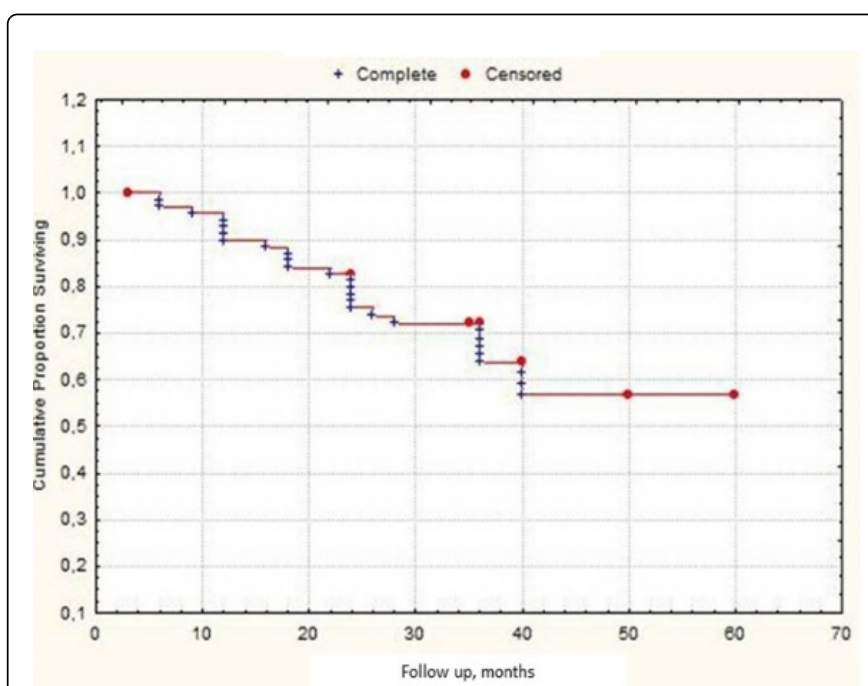

Figure 3: Overall efficacy of catheter treatment of post-infarction ventricular tachyarrhythmias.

It is assumed and demonstrated in several studies that ventricular tachyarrhythmias elimination reduces the rate of ICD interventions, amiodarone usage and hospitalization, which should lead to clinically relevant improvement of the life quality $[7,8]$. It meets the patients' needs and reduces the long-term costs. 
Page 5 of 6

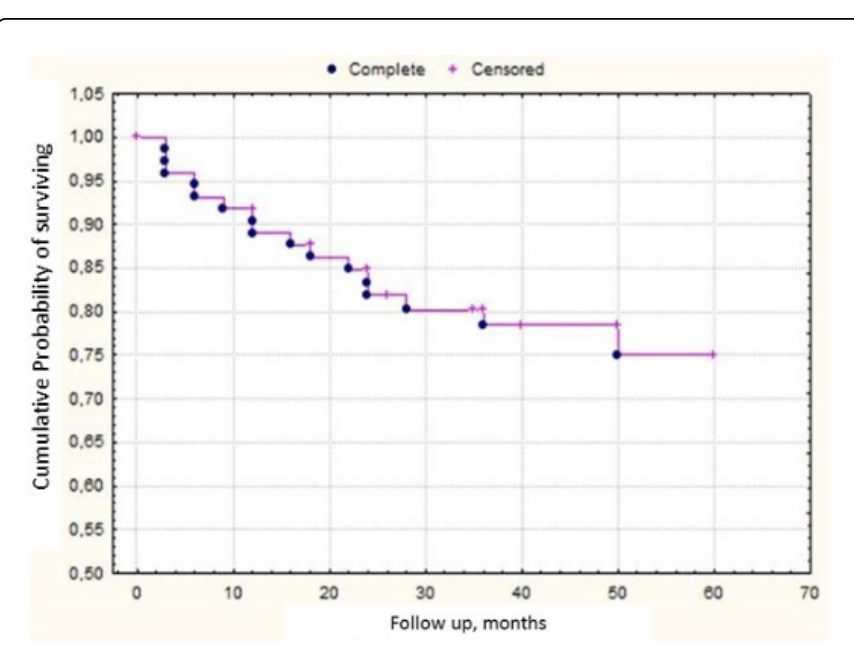

Figure 4: Survival rate after catheter treatment of post-infarction ventricular tachyarrhthmias.

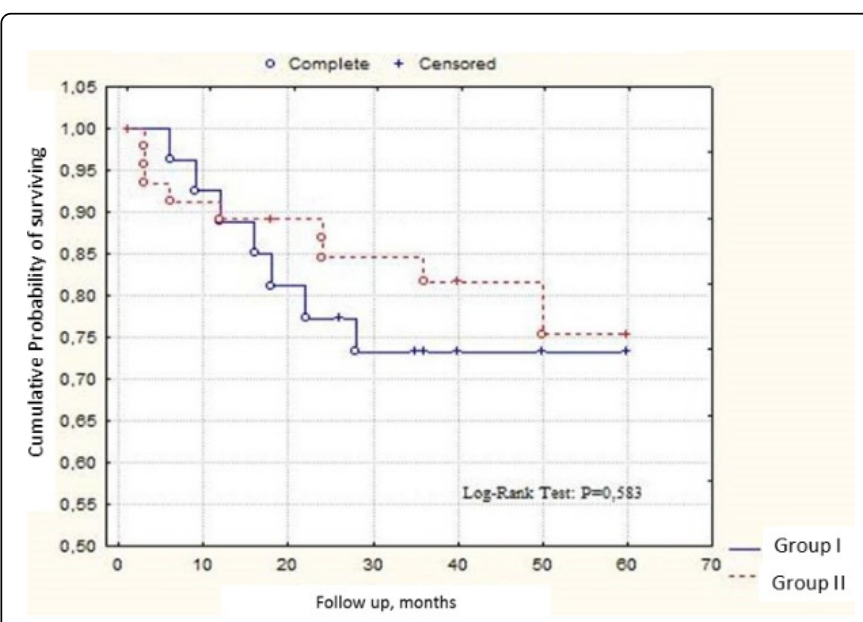

Figure 5: Survival rate (all-cause mortality).

Nevertheless, according to the data acquired, the postponed efficacy results demonstrate that successful elimination of ventricular tachycardia can have no influence on the patients' survival. Recurrent ventricular tachyarrhythmias after catheter ablation are not unexpected, taking into consideration the complex substrate of ventricular tachycardia, thus the results are far from being ideal. Even with sufficient intraoperative ablation success, ventricular tachyarrhythmias can arise repeatedly due to several mechanisms including progressive remodeling of existing scar field with development of new re-entry cycles or change of the exit point of the previous clinical tachycardia after prior catheter ablation.

Even with the suggested method of complete elimination of abnormal potentials, which should prevent the emersion of new reentry circles, the incomplete transmurality of lesions diminishes the procedural success.

The high rate of postoperative mortality, mostly due to congestive heart failure decompensation, is dismal but not unexpected. It is known that recurrent adequate ICD shocks in such patients can lead to 2-5 times multiplication of mortality rate due to progressive heart failure. The SCD-HeFT study results assume, that myocardial lesion after the ICD shock is unlikely to become the mechanism of systolic function depression, and apparently deterioration of arrhythmia indicates the progression of heart failure.

It is worth noting that multidisciplinary approach to heart failure treatment was applied to all participants. All patients took the optimal drug treatment, device follow up was performed regularly to avoid the right ventricular stimulation, also atrial tachyarrhythmia was controlled. Such approach is necessary to reach the optimal long-term results of ventricular tachyarrhythmias catheter ablation. In spite of these positive results, the rate of arrhythmia recurrence (40\%) and mortality $(22 \%)$ remains high and correlates with the previous clinical reports and studies. The specificity of our study is the longer follow-up period of 5 years, while generally it is limited to 3 years [4].

Now-a-days interventional electrophysiology set the goal to hold multicenter and randomized clinical trials to evaluate efficacy and survival in patients with ventricular tachyarrhythmias undergoing ablation compared to standard approach without catheter treatment.

However the range of patients with ventricular tachyarrhythmia is wide and it is difficult to evaluate the real clinical efficacy of catheter ablation or its influence on survival in this population. It would be better to compare separate groups divided by: age, left ventricle ejection fraction (e.g. level of $20 \%$ and less vs $30-35 \%$ ), history of electrical storm, functional class of heart failure etc.

\section{Conclusion}

During 5 years of follow up the overall efficacy of catheter elimination of ventricular tachyarrhythmias and patients' survival was $63 \%$ and $78 \%$ respectively. The was no statistically significant difference in mortality rate in patients with effective catheter treatment and those with recurrent ventricular tachyarrhythmia after RFA.

\section{Study limitation}

The patients differed in time of ventricular tachycardia onset after acute MI whereas some of them had different types of reperfusion. It is important that study participants underwent only endocardial ablation, without systematic epicardial approach, which obviously could influence the catheter treatment efficacy. There was no evaluation of the recurrent hospital admissions rate, quality of life, ICD interventions and cost-effectiveness of treatment after catheter ablation.

\section{References}

1. Huikuri HV, Castellanos A, Myerburg RJ (2001) Sudden death due to cardiac arrhythmias. N Engl J Med 345: 1473-1482.

2. Connolly SJ, Hallstrom AP, Cappato R (2000) Meta-analysis of the implantable cardioverter defibrillator secondary prevention trials. AVID, CASH and CIDS studies, antiarrhythmics vs Implantable defibrillator study, cardiac arrest study Hamburg, Canadian implantable defibrillator Study. Eur Heart J 21: 2071-2078.

3. Bardy GH, Lee KL, Mark DB (2005) Sudden cardiac death in heart failure trial I. Amiodarone or an implantable cardioverter-defibrillator for congestive heart failure. N Engl J Med 352: 225-237.

4. Poole JE, Johnson GW, Hellkamp AS (2008) Prognostic importance of defibrillator shocks in patients with heart failure. N Engl J Med 359: 1009-1017.

5. Tung R, Vaseghi M, Frankel DS (2015) Freedom from recurrent ventricular tachycardia after catheter ablation is associated with improved 
Citation: Garkina S, Tatarskiy R, Lebedev D (2018) Survival in Patients with Ischaemic Cardiomyopathy and Ventricular Tachyarrhythmias: Role of Catheter Treatment. J Cardiovasc Dis Diagn 6: 336. doi:10.4172/2329-9517.1000336

Page 6 of 6

survival in patients with structural heart disease: An international VT ablation center collaborative group study. Heart Rhythm 12: 1997-2007.

6. Sapp JL, Wells GA, Parkash R (2016) Ventricular tachycardia ablation versus escalation of antiarrhythmic drugs. N Engl J Med 375(2): 111-121.

7. Tatarskii RB, Lebedev DS, Lebedeva VK (2015) Extrennaya cateternaya ablaciya electricheskovo shtorma u bolnikh s implantirovannimi cardioverterami-defibrillatorami. Rossiyskii cardiologicheskii zhurnal 11: 57-62.

8. Marchlinski FE, Haffajee CI, Beshai JF (2016) Long-term success of irrigated radiofrequency catheter ablation of sustained ventricular tachycardia: Post-approval thermo-cool VT trial. J Am Coll Cardiol 16: 674-683. 